\title{
The Characteristics of Language in Cosmetic Advertisements
}

\author{
Zhihong Bai \\ Shanxi Normal University, Linfen, China
}

\begin{abstract}
Advertising plays a vital role in the development of society and economy. Its function can not be underestimated. Advertising language is a special applying language when it compares with others like literary and technological languages. It has become an indispensable part of daily life because of its particular characteristics. It makes a brief introduction into the definition, function of advertising at first in this paper. Then, it mainly summarizes and analyzes the linguistics characteristics of cosmetic advertisements at lexical, rhetorical levels and sentence structure. Through the analysis of cosmetic advertisements, the linguistic features of advertisements are summarized. It is important for people to understand the beauty of language in advertisements.
\end{abstract}

Index Terms—advertisement, cosmetic advertisements, linguistic characteristics

\section{INTRODUCTION}

Advertisements play an important role in people's daily life. We can see the advertisements on TV, on the newspaper, on the internet, in the movie even on the buses. Advertising has pervaded in human's daily life. Everyone can see or hear many advertisements everyday. The market share of one particular cosmetic brand has much to do with the effect of advertisements. Manufacturers take advertisements as one of the most powerful "weapon" to open the market and promote their products. Advertising is beneficial for manufacturers to increase profits, manage attitudes and social values. It also makes great contributions on shaping people's life style. Moreover, advertisements influence our language, culture and psychology.

Advertisements are made up of three types of information: audio, optical and language. Usually, audio, language and visual collectively function in promoting the brand. In the writer's opinion, the information given by language is more exact. For this reason, this paper will introduce the linguistic characteristics of cosmetic advertisements at lexical, structure of structure, rhetorical. There is a literature review at the beginning. In the end, there is the conclusion. The key of this paper is the middle of three parts, that is, respectively analyzes the linguistic characteristics of cosmetic advertisements at lexical, the structure of sentence and rhetorical levels. From the data analysis, the writer provided the conclusion of the thesis.

\section{LITERATURE REVIEW}

In this part, the basic concept of advertisements will be discussed first. It contains the definition and role of advertising, the meaning of cosmetic advertising language will be introduced as well. In the last part, the studies on cosmetic advertisements and cosmetic advertising language at home and abroad will be reviewed.

\section{A. Definition of Advertising}

The word "advertising" originate from the Latin word "adventure", which means to catch the public's attention and lead them to certain direction, the advertisements become fashionable since the end of the 17th century. Advertising is "a form of communication for marketing and used to encourage, handle or convince listeners (sometimes a specific group) to continue or take some new actions. Although ideological and political advertising is also usual". Another well known definition is "the non-personal communication, which is usually forceful and paid for in nature about commodities, services or ideas by specific organizers though the variety of media" (Zhaojing, 1991, p.1). These two definitions share some similarities: Advertisements are non-personal linguistic form. They contain mass media. It is used to communicate information or persuade potential audience.

The definition mentioned is related to commercial advertisements, which is one classification of ordinary advertising. Another classification of advertising is public service advertising. Even though public service advertising also values a lot in the society, it isn't discussed in this paper. Instead, commercial advertisements, especially the cosmetics advertisements will be discussed in this paper. It includes foundation makeup, body cleanser, sun screen, facial cleanser, puff cake, perfume, toner, lotion, cream, and so on. Because of the particular characteristics of cosmetic advertisements, there are differences between cosmetic advertising language and other types of advertising language.

As a special kind of speech behavior from the definition of advertising, the purpose of advertising is to encourage the audience to continue or take some new action. Persuading and informing are two main functions performed by advertising (Crystal and Davy, 1983). Many scholars hold the idea that the two functions of advertising are not of equal 
importance. People use advertising to present certain information about their goods to the public, in order to persuade the audience to make choices or take actions. The purpose of presenting message of advertiser is not for expending audience's knowledge, but a preliminary for the function of persuasion. The advertisers need to consider the needs, income, psychology, gender, culture when they pass the information to the potential consumers. That's the final purpose of advertisements. The language in an advertisement must be able to "attract attention, motivate desire, evoke interest, create conviction and get action" (Vesterguard\&Kim, 1985, p.49).

\section{B. The Definition of Cosmetic Advertising Language}

The word "cosmetic" derives from the Greek word "kosmetikos". And it firstly appeared in 1605. When we relate to the cosmetic products, many kinds of products such as day cream, make-up, night cream and so on. But they aren't enough. According to Webster's Third New International Dictionary (1993, p.514), cosmetic is defined as "a preparation(except soap), preserving, or changing the appearance of a person as for conditioning, cleansing, coloring."

The aim of the study is combining the previous definitions of advertising and cosmetics, cosmetic advertising language can be defined as follows, "As a special one type of advertising, cosmetic advertising language can be a non-personal communication of information between the potential consumers and advertisers (especially women), which includes the title and contents of cosmetic advertising and aims to make their cosmetic brand, products and service known to all and urge the consumer to buy their products through the media." The basic function of cosmetic advertising language is providing some information about products, persuade to make choices and take some actions at last, peculiarly to buy products.

\section{The Research Situation at Home and Abroad}

The advertising industry is prospered in England at the end of $17^{\text {th }}$ century. After that, scholars are interested in this area. The English linguist Leech studies the characteristics of advertising language from the view of semantics. Vesterguard and Kim (1985) studies advertising language from the view of macroscopic. The research about advertising language at home appeared in the late 1980s. Qin Xiubai(1986) studies advertising language from stylistics. Huang Guowen (2002) studies advertisements in discourse level. There are also scholars who studies advertisements in economic area or psychological point of view.

When Huang Guowen published his Problem-Solution Pattern in Cosmetic Advertising in 1997, the study of cosmetic advertisements caught scholars' eyes quickly. Besides, many scholars are interested in cosmetic advertisements and fruitful researches are produced from other various fields. The earliest cosmetic advertisement is produced by the American cosmetic company. The same as other varieties of advertisements, conventional structure of cosmetic advertisements contain a headline, the main part, company information and the brand name. More and more cosmetic advertisements cover two or more elements we mention above. There is one common characteristic of cosmetic advertisements: take the female as the target audience. Williamson believes the cosmetic advertisements not only to promote the products to the female consumers and their expectation but also have a more attractive image.

Comparing with other types of advertising language, cosmetic advertising language has its own unique features. Firstly, the female features in the language are obvious. For example, pursuing of nature, beauty, safety and so on. Secondly, the advertisers use adjectives to meet the demand of the potential consumers. At last, there are many color words in cosmetic advertising language. According to the research, we find that like the other special linguistic forms, cosmetic advertising language has its unique features in structure of sentence, lexical levels and so on. In summary, cosmetic advertisements are one type of advertisements, spread some information to the potential consumer and its ultimate goal is to convince them to buy cosmetic products through some medias.

\section{RESEARCH DESIGN}

This paper analyzes the linguistic characteristics of cosmetic advertisements at lexical, the structure of sentence and rhetorical levels reference to former research findings and survey methods respectively to provide the language of cosmetic advertisements for a certain help. It includes research purpose and research methods.

\section{A. Research Purpose}

A successful advertisement is to make consumers want to buy, so to attract consumers, advertisers must carefully consider the language of advertising. In fact, the success of an advertisement does not lie in the application of a certain factor of voice, vocabulary, grammar and pragmatics. It is often a combination of factors that can create a successful advertisement. So this thesis is carried out from vocabulary, grammar, syntax, pragmatics and other aspects to make some contributions to achieve the success of the cosmetic advertisements.

\section{B. Research Methods}

Firstly, the literature method is used in the research, through the classification of the related literature. Secondly, the way of classification through mastery of content about topics, and some data collection and collation are used in the research to make the whole process of classification. Thirdly, the action research method is used in the survey, it tries to classify all kinds of cosmetic advertisements, which generalizes the characteristics from the aspects of vocabulary, the structure of sentence and rhetorical levels, then continue to explore and summarize to draw general conclusions. 


\section{The Analysis of Characteristics of LANGUAGE In COSMETIC ADVERTISEMENTS}

This part will introduce the characteristics of language in cosmetic advertisements from vocabulary, rhetoric and sentence structure aspects.

\section{A. The Vocabulary in Cosmetic Advertisements}

Nowadays, the pace of living is becoming quicker and quicker with the development of technology. However, few people is willing to slow down to read a piece of advertisement carefully. The advertisements in our lives are usually composed of only a few words. As a fashion industry, vocabulary in cosmetic industry has its unique features.

\section{The Use of Adjective}

In the study of cosmetic advertisements, adjective appears mostly in cosmetic advertising language. That is to say, adjective is an indispensable part for cosmetic advertisements. Adjective is the core. The examples will be listed as the following:

(1) Expose your glamorous side, your stunning, luminous, brunette goddess side. (Shampoo Advertisement)

(2) Discover a dermatologist's way to reveal fresh, new, healthy skin. (L'Oreal Advertisement)

(3) Lip so moisturized, they can silky, soft, smooth. (L'Oreal Lip Color)

Obviously, these cosmetic advertisements contain a high proportion of the adjective than general English article. Some are habitual collocation, these adjectives rarely appear in daily spoken English. For instance, "smooth, silky, soft, fresh, new, healthy" often appear in the skin cream advertisements, "fragrant, fresh, pure" always can be found in the perfume advertisements. Nearly everyone of us can bolt out two or three adjectives used in advertisements. This is still determined by the function of advertisements--to promote the brand. Therefore, at least it should let consumers know two things: what is the product and how is the product. Furthermore, adjectives used in cosmetic advertisement are usually at the superlative degree. There is an example for this: Defend your blonde from the attack of every day residue and stay your lightest and brightest, your best blonde ever. (Sheer Blonde Shampoo Advertisement) We know that light and bright should be the common feature of shampoo. In order to show the advantage of the present similar products, the advertiser accepts adjectives in the highest level.

\section{The Use of Verbs}

In the study of the verb of cosmetic advertising language, a significant observation shows that as long as it doesn't affect the understanding of this whole article, cosmetic advertiser don't use verbs. There are many cases of cosmetic advertising show that verb almost be omitted. The examples will be listed as the following:

(1) Sheer brilliance. A shiny kiss of crystal color. (Channel Lipstick Advertisement)

(2) Fragrant as fresh-peeled Lime, fresh as nature itself, frothy lure lime shampoo. (Lime Shampoo Advertisement)

There is a common feature in cosmetic advertisements, that is, verb doesn't appear in the advertisements. Verbs simple using is another significant feature in cosmetic advertising. The book of the famous British linguist Geoffrey Leech once summarized the top-pest 20 verbs used in cosmetic advertisements, they are make, protect, discover, build, provide, feel, show, help, clean, love, keep, wear, wash, bring, give, use, take, look, need, like. All of them represent an actual action and are easy to understand and remember. This is resulted from the function of advertisements. Cosmetic advertisements are the ways of publicizing and promoting a brand, which means it should make consumers catch the focus meaning at their first sights. If the word used is so unfamiliar that the reader needs to ask a dictionary for help, the advertisements limit the range of potential consumers and fail to reach the desired effect.

\section{The Use of Phrase}

The noun phrase with synthesis of modifiers and verbal phrase is simplified. Some verbal phrases still include more than one word, on this occasion, the advertisers often use the following structure: verb+object+object complement. This center word of the structure is a simple verb. Although the function of verbal phrase in cosmetic advertisements is very important, verbal phrase doesn't described in this paper, it mainly considers the function of noun phrase and different functions of adverbial phrase in the process of communication.

\subsection{Noun Phrase}

The complexity of noun phrase is one of the significant features of cosmetic advertising language. Cosmetic advertisers try to use the minimum number of words to describe the products and service. This goal is mainly achieved by noun phrase. A center word and multiple modifiers often appear in cosmetic advertising language. For example: fast-foaming, quick-acting shampoo; natural, a fresh, healthy-look pressed powder and water-based liquid makeup. It is worth noting that the noun phrase in the cosmetic advertising language loaded with multiple modifiers. Although the excessive modification of noun phrase can catch the reader's attention, the concentration can not be quickly understood.

\subsection{Different Functions of Adverbial Phrase in the Process of Communication}

Adverbial phrase is not as important as other phrases, but also take the most seat in cosmetic advertising language. It usually expresses a certain attitude, place, time, purpose and result. Adverbial phrases can be divided into four different forms: 1) conjunctive adverbs, such as "because, even if, why, when" and so on. 2) adverb phrase, such as "feeling lovely and cheerfully, stay fresher, taste so good" and so on. These adverbs are similar to the adjective, virtually play the role of verbs. 3) because of its play to the function of adverbs, so these adverbs with prefix is called adverbial phrase. 4) collective adverbs, such as "every day, any time".

\section{B. Rhetorical Analysis of Cosmetic Advertising Language}


As a major kind of linguistics, rhetoric is a useful and interesting way to convince people. The cosmetic advertising language is a quite forceful language. Therefore, there are a lot of rhetorical devices in cosmetic advertisements. The suitable use of rhetorical devices is sure to enhance the artistic and strengthen the expressiveness. Now, the writer will appreciate these wonderful sentences in cosmetic advertisements.

\section{The Use of Metaphor}

In the cosmetic advertising language, metaphor is mainly used to improve the convince of information. A metaphor makes comparison to describe a word or phrase is same as another things. It is another way of comparison. The comparison in metaphor is a more implicated way, people need to use more wisdom to find the beauty of metaphor. The examples will be listed as the following:

(1) A perfect storm. And in body copy it suggests: Max-wear Lip color makeup that a weathers storm and create one too. (Max Lipstick Advertisement)

(2) More moisture, and what a relief--same addictive feel. The signature line is "never go thirsty again" (CLINIQUE Skin Cream)

In the example (6), the advertiser links the lipstick to the implied meaning of storm. More interesting is the word "storm" appear three times in the advertisement, it is quite a perfect storm, weather storm, create one,. This advertisement can be understood as Max lipstick can protect lips from weather. On the other hand, Max attracts the opposite sex. The example (7) is an advertisement of skin cream. In this case, the example implies that the skin without moisturizer feels like thirsty. Metaphor is widely used in perfume advertisements. For instance, "A seductive gathering of lush fruits and sumptuous flowers." The word "seductive" is interpreted as perfume is attractive, which draw the interest of potential audience.

\section{The Use of Metonymy}

Metonymy is substitution of one thing by another. Metaphor and metonymy play important roles in linguistics, which is substitution of one word by another word. However, these two kinds of rhetorical devices play different roles in linguistic. Metonymy mainly refers to the similar of different things, and metaphor mainly refers to the connection between the two. The appropriate use of metonymy makes the image more impressive. The examples will be listed as the following:

(1) Head \& Shoulders Extra Fullness is a dream.

(2) Advanced Radiance beats that lost-sleep look by instantly softening appearance of fine lines and shadows and helping brighten skin for a look that's five years younger!

(3) Thank goodness for Head \& Shoulders Ultimate Clean.

These examples are cosmetic brand name, but it is sure to represent the cosmetic products. Brand name and product connect in a domain. This is the traditional application of metonymy in cosmetic advertising language. Except for the general metonymy, people can find creative metonymy in cosmetic advertisements. For example, a perfume advertisement for male "Backham fragrances--a new fragrances for man". David Beckham is a excellent football stars, he represents the charming. In this advertisement, "Baceham" is a brand of perfume, and it represents a kind of perfume.

\section{The use of Personification}

Personification makes the things which don't have the behavior and emotion that people have be same as people. Examples include ascribing human emotions to force of nature and describing jinn with human form. The use of personification can perk up the cosmetic advertising language and make it more vivid and attractive. The examples will be listed as the following:

(1) With OLAY mousse Cleansers you can also moisturize or refine the look of pores, even fight aging appearance.

(2) Your skin will love to drink it.

(3) Hair color remover, So lighten up and have fun with your hair.

(4) CLINIQUE will tell you something scientists know--UVA protection is important, but just no longer enough to prevent signs of aging.

(5) Promise your nail a new future.

After reading these examples, the characteristics of cosmetic advertisements can be found, they are endowed with actions as human being. For example, "OLAY can fight aging appearance", "have fun with your hair", "CLINIQUE can tell you something" and so on. In addition, there are some human behavior and emotion are also used in cosmetics advertising language, such as "skin love to drink it", "nail has a new future". Through the personal way, it makes advertisements more interesting and helps people to create personalized products. Thus makes the personal products more acceptable.

\section{The Use of Parallelism}

Parallelism sentences have used to express a same idea in English and Chinese languages, emphasize the language potential, emphatic rhetorical devices. It is such a ordinary literary device. And it is hardly ever noted as a figure of speech. And this device is widely used in cosmetic advertisement to strengthen people's impression towards certain brand and features of its product. The examples will be listed as the following:

(1) I will never forget her kiss, her smile, her perfume. (Channel No.5)

(2) As real as breathing, truth is the only word to be remembered, truth is a free face, truth is the perfect after 
deposition. (Sofnon Advertisement)

These advertisements use parallelism and the advertisers use simple words over and over again to achieve the effect of easy to remember, give their consumers deep impression. Such as, the word "her" appears several times in the advertisement to enhance people's impression towards their products. It is easy to appear the advertisements in people's brain when they choose and buy the product. By this means, advertiser improve the competitiveness of their products in the same type of products. This is a very powerful "weapon" to promote their products.

\section{The Use of Pun}

The pun is a way of word that shows the meaning of two or more by using plenty of meanings of words, in order to achieve rhetorical function and intended funny. Puns may be identified as conventional structure, given that their meaning is totally local to its culture and a particular language. The same sentence may have two different meanings, however it represents expression. That is to say, puns need many words and phrases to understand. Puns used by comedy writers for a long time. And in cosmetic advertising, it also claims a special manor. The examples will be listed as the following:

(1) Dior addict. ( Dior Lip color Advertisement )

(2) Enhance the eyelids, smoothing deep wrinkles, flashing big eyes. (Eye Cream)

(3) Be fair to your complexion. (Dermacare Whitening Cream)

(4) Who cares if your hair is different? Sunsilk care with three special shampoo. (Sunsilk Shampoo Advertisement)

In the first example, addict has two meanings. On the one hand, it introduces the function of the lip gloss--attract the opposite sex. On the other hand, it highlights the features of product, only a can of Dior make you addictive, other lip gloss can not. In the second example, the word "big" has two meanings. One is the meaning of the original word and the scope is at a very high level. The other is the meaning of adjective, that is, compared with "small". And in the example (19), "big" means eyes become bigger after use the product. The use of the word not only perks up the cosmetic advertising language, but also helps the consumers know the function of product to achieve desired result. In the fourth example, using the word "care" has two different meanings. The first word "care" is a verb. The second "care" is a noun.

\section{The Use of Rhyme and Alliteration}

Rhyme is a repetition of the same sound or similar sounds in two or more words, usually on the last syllable in poems and songs. And the cosmetic advertisements also use the rhetorical device. Rhyme serves as a powerful device to remember. And rhyme is pleasant to hear. The use of rhyme helps to mark the end of the syllable. And the listener knows the syllable structure. Advertisers use rhyme in their advertisements to draw the attention of potential consumers. Besides, the advertisers use alliteration in their advertisements to achieve the function of persuasion.

Alliteration derives from the Latin word "litera" meaning "letters of the alphabet". Alliteration has beautiful effect. And alliteration is not only used in poetry, but also in the title of book especially in cosmetic advertisements. The use of alliteration in cosmetic advertisements will strengthen customers' impression of the products and persuade the audience to make choice or take action. The examples will be listed as the following:

(1) A piece of soap is so special, it's made for your face being a smooth silky skin.(Soap Advertisement)

(2) Avon-- the more beautiful shop in town.

(3) Minimize lines; maximize your savings.

(4) Slip, Slop, Slap. (Slip on a shirt, sloping on some sun-screen lotion, and slapping on a hat.)

In the example (22), the use of alliteration are "a soap so special" and "a smooth silky skin", it emphasizes the characteristics and function of product. In the example (23), as one of the world's most prominent cosmetics company's advertising, it has already known very well by us. The use of two "s" shows the position of the company and highlights the features of company. In the example (24), the aim is essentially to promote product at lower price. In the fourth example, advertisers persuade consumer use the product to protect their skin from UV rays.

\section{The Sentence Structure in Cosmetic Advertisements}

It is obvious that word is not enough to make a piece of good advertisement. Another feature of cosmetic advertisement is its sentence structure. The sentence structure in cosmetic advertisements tremendously differs from that used in other estrogen and situations such as in novel, prose and drama.

\section{The Use of Primitive Sentence}

It is sure that a sentence that can occur in our mind at any time and any place must be a short and simple sentence. According to a scientific research, man can quickly grasp the focus meaning of a sentence and repeat it shortly after listening to it when it is formulated by less than 24 words. If we have a glimpse over the sea of cosmetic advertising, we can find that the most of them are only a line of words. The examples will be listed as the following:

(1) Lime--fragrant,fresh,pure.

(2) After cleansing,smooth on,rinse off,like conditioner.(OLAY Body Lotion )

(3) Leave pores detoxified, leaves skin purified.

(4) For the new dew look Nivea vanishing cream with eucerite.

(5) No wonder, Neutrogena is recommended most by dermatologist.

These are representative simple sentences. The kind of expression is extremely concise. In present society, no one will spend an extra period of time analyzing and understanding what an advertisement wants to say and this requires a 
sentence in the advertisements to be short. The characteristics of cosmetic advertisements are closely related to its function. And another reason why the expression in advertisements is concise lies in the high cost of a piece of an advertisement. Pick up the TV advertisement as an example, a company has to pay millions yuan for a second and this thus force the expression in advertisements to be concise and effective.

\section{The Use of Imperative Sentence}

In our daily life, imperative sentences are used for suggest, ask or order others to do something and recommend. And this feature of imperative sentence meets the demand of advertisements which aims to make consumers pay for the product. At the same time, imperative sentence also features for being able to cut the point directly and make consumers know its meaning within several seconds. Therefore, there is no wonder that we can meet many imperative sentences in the world of cosmetic advertisements. The examples will be listed as following:

(1) Come experience the word...As you've never experienced it before...(OLAY)

(2) Don't be afraid. Don't worry about travel in summer. (Nivera)

(3) Perfect you most extraordinary eye-catching blonde. Daily use refreshes and perfects treasured highlights, revitalizing all shade blonde.

(4) Let skin young twice a day

These examples show that the advertisements bring a cordial feeling to us. Our hope and desire to enjoy product or service will be evoked immediately. Words and phrases like let's, enjoy and go ahead possess tremendous power of calling for people to take actions. This is useful strategy to promote sales.

\section{The Use of Question}

The natural curiosity of people drives them to pay more attention and think more deeply when they meet something they do not know. And if the answer is showed to people finally, it will usually stick in their brains. And the use of question takes the advantage of this feature. Firstly, it throws a question to you deliberately and wakes up your curiosity, and then it gives you several sentences to promote the product. The examples will be listed as the following:

(1) Is her skin really this beautiful? Don't you love being a woman? Max Factor.

(2) Who cares if your hair is different? Sunsilk cares with three special shampoo.

(3) Why your skin drink it down so quickly? (OLAY)

These examples show that the advertisements wake up our curiosity. The first example lists a group of two questions to catch the attention of woman. Firstly, it lists a question "Is her skin really this beautiful?" Secondly, it continues to ask question, then it answers the question. Max Factor makes people have beautiful skin. Each woman wants to have beautiful skin, so they get a lot of attention to keep beauty. Under these circumstances, woman will buy and use the products. The example (37) is a very simple question, nevertheless, it contains much implication. Please note the word "quickly", which means the function of the products. And then she will wonder that what is the difference between this one and other similar products, what kind of new technique is used in the product, at the end of these questions she will want to have a try on her own and answer these questions by herself. This is a useful strategy to attract consumers' attention.

\section{CONCLUSION}

Cosmetic advertising language, as a rapid development of fashionable jargon, has affected many consumer's consumption concept and buying behavior. The rapid development of cosmetic advertisements demands in-depth researches in this area. By analyzing the lexical, structure of sentence and rhetorical levels of cosmetic advertisement, the writer finds out the characteristics of cosmetic advertising language. Based on the previous discussions and analysis, the major findings in this paper will be briefly summarized in what follows.

The advertisement is a kind of text which combines informative function, expressive, aesthetic and vocative functionality in one. Its multiple functions determine that cosmetic advertising language has its own features. Along with the analysis of the paper, the writer finds that cosmetic advertisements make the language concise, interesting, more informative and euphemistic. Those aspects are the embodiment of the functions in cosmetic advertising language, with which the language could be more persuasive and attractive. And the cosmetic advertiser could use more effective advertising language to promote their products. Meanwhile, the proper cosmetic advertising language could benefit the consumers in the following aspects: get more useful information within the limited time or space, enjoy this special kind of communication.

The paper on the whole makes a deeper research. Efforts have been made to find out how the cosmetic advertising is extraordinary through the analysis of three aspects: lexical, rhetorical devices, sentence structure. At lexical level, simple words that show the functions of cosmetic is preferred. Besides, other ways of word formation such as phrase are also widely used in cosmetic advertisements. At rhetorical device, it plays an important role in making the words more beautiful. At syntactic level, in order to make the expression more short and concise, simple sentence takes the most seat in cosmetic advertisements. Question, imperative sentences are also adopted to achieve certain effect.

In conclusion, cosmetic advertisements, as a specific genre or discourse, can show many things, such as ideological and many cultural features as well. This investigation is only tentative attempt. As the ability of the author is limited, the data collection also has certain limitation, so the weak point herein is unavoidable. It is requested that earnestly fellow teachers to give the criticism to point out mistakes. 


\section{REFERENCES}

[1] Crystal, D \& Davy, D. (1983). The Cambridge Encyclopaedia of language. Cambridge: Cambridge University Press.

[2] Huang Guowen. (2002). Theory and Practice of Discourse Analysis-Advertising Discourse Research in Advertising. Shanghai: Shanghai foreign language education press.

[3] Qin Xiubai. (1986). Introduction to Stylistics. Changsha: Hunan people's Publishing House.

[4] Vesterguard, T and Kim, S. (1985). The Language of Advertising. India: Basil Blackwell Publisher Ltd.

[5] Zhao Jing. (1991). English for Advertising. Beijing: Foreign language Teaching and Research Press.

Zhihong Bai was born in Lvliang, China in 1993. She is studying for her Master's degree in linguistics in Shanxi Normal University, China.

She is currently a student in the school of Shanxi Normal University. Her research interests include translation and applied linguistics. 